\title{
THE HST OBSERVATIONS ARCHIVE AT ST SCI
}

\author{
Ron Allen \\ Space Telescope Science Institute \\ Baltimore, MD 21218 \\ U.S.A.
}

1. All data generated by HST are preserved in a specially-designed interim archive system, the "Data Management Facility" at ST ScI.

2. Data is currently being written to $2 \mathrm{~Gb}-12$ " optical disks at the rate of about 0.5 $\mathrm{Gb} / \mathrm{day}=35$ exposures/day.

3. A catalog is produced as the data is archived. This catalog is publically accessible and currently contains information on over 19,000 exposures (images, spectra, flat fields, calibration data, etc.)

4. About 14,000 exposures are now non-proprietary; more than a third of these are observations of sky targets obtained during OV/SV.

How to obtain information about the HST Archive:

1. By telephone: (301) 338-4413 User Support Branch (USB) or 1-800-544-8125 (tollfree in the USA)

2. FAX: (301) 338-5085 User Support Branch

3.e-mail: usb@stsci.edu (Internet)

or: usb@stsci.bitnet (BITNET)

or: STSCIC::USB (SPAN)

4. STEIS: "anonymous ftp" to stsci.edu (Internet 130.167.1.2) See especially the directory "observer/completed_observations" for the Archived Exposures Catalog.

\section{Obtaining HST Data from ST ScI}

Accessing the catalog:

1. Browse the HST catalog with STARCAT.

A "Quick Guide to STARCAT" is available from the User Support Branch at ST ScI. To run STARCAT you must "telnet" to "stsci.edu" 
2. Retrieve your list of marked data sets from "stsci" with "anonymous ftp". Your file is in the directory "starcat".

Obtaining your data:

1. Complete the request form (you got this with the STARCAT Quick Guide), and mail it to USB at ST ScI.

2. Requests for small amounts of data (e.g. two tapes or less) will be filled \{lem gratis \} on a level-of-effort basis.

3. Larger amounts of data will require re-imbursement for media and copy costs.

4. Financial support for Archival Research is available through the normal HST proposal process. Contact USB for information. 